\title{
Detection of Coverage Hole Nodes in Wireless Sensor Network using Artificial Intelligence
}

\author{
Mainka, Khushal Thakur, Kiran Jot Singh
}

\begin{abstract}
Adequate coverage of the sensing field in Wireless sensor networks (WSNs) is critical to many applications. However, when one or more sensor nodes stop working due to energy exhaustion or physical damage, the network may experience overlay vulnerability. This can disrupt network connectivity and hinder performance. Therefore, it must be fixed automatically. To resolve this problem, swarm inspired Artificial Bee Colony $(A B C)$ scheme in addition to the Artificial Neural Network (ANN) approach is used. The aim of ABC is to optimize the shortest path by selecting an appropriate fitness function and then identify holes using ANN. Before the detection of holes, ANN is trained as per the optimized properties of nodes that are as per the genuine nodes and coverage hole repair properties. Therefore during the testing process, ANN compares these properties with the stored properties and then identify the hole repair node. From the experiment, it has been analyzed that the energy consumption up to $23.88 \%$ is saved.

Index Terms: WSN, coverage holes, mobility, $A B C, A N N$
\end{abstract}

\section{INTRODUCTION}

A WSN consists of tiny sensor nodes, each of which is used to sense certain physical properties, performing some task on the collected data and communicate with each other [1]. The sensor nodes are installed in a remote area with multiple numbers and interconnected to create a network known as WSN as shown in figure 1. All the sensor nodes are self-organized, each of which is responsible for the data collection and transformation using the sensor node named as a source node and destination node respectively [2]. These networks find application in various fields such as in civil and military application. In civil applications such as used for environmental monitoring, traffic control whereas in military WSN can be used to identify attacker, to track objects and disaster recovery [3].

To collect data from the sensor nodes deployed in these applications needs adequate coverage of the sensing area so that the sensed data can be transmitted to the receiver node [4]. The observation of sensing area helps to measure the region, which is being monitored by the deployed nodes [5]. Therefore, nodes must be deployed either randomly or in a planned way lies in a region of interest (ROI). The network faces the problem of coverage holes, in case, when the nodes lose their energy or any hardware failure. After the appearance of coverage holes the network stop functioning or that area cannot be monitored by the sensor nodes.

Revised Manuscript Received on June 15, 2019.

Mainka Nafri, Khushal Thakur Kiran Jot Singh, Chandigarh University, Mohali, India.

In addition, the formation of coverage holes is a predictable phenomenon in WSN, and if it remains unattended, the number and size will gradually increase [6]. Covering the existence of vulnerability reduces and destroys the functionality as well as the throughput of the complete network.

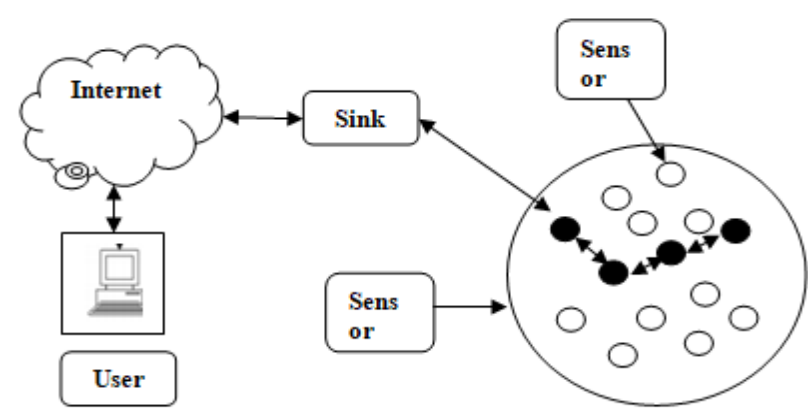

Figure 1 WSN architecture

The coverage holes can damage data transfers, disconnect the network connections, and leave certain areas of the network unmonitored. Therefore, one may not be able to observe critical events or may compile inaccurate data [7]. As the continuous monitoring of the network is very important, which again depends upon the sensing of field therefore, it is necessary to make sure that the sensing area must be covered continuously. Since, the network is designed to a remote area where the human approach is unfeasible. Therefore the management of WSN connectivity becomes a serious problem. Therefore, we must design a network that can detect and repairs these holes with high efficiency and at the accurate time [8]. In this research, to resolve this problem, optimization technique along with artificial intelligence has been used.

\section{RELATED WORK}

Sahoo et al. $(9,2015)$ presented an effective hole repair algorithm by using the concept of reducing the coverage area of overlapping nodes. This helps to keep nodes connected with the limited movement of the nodes. The nodes are selected as per the rate of overlapping coverage area. The mobility of the nodes has been limited by one hop and hence save the amount of energy. Zhao et al. (10, 2016) have focused on detecting boundary holes as well as coverage holes.

Two algorithms named as Distributed Sector Cover Scanning (DSCS) and Directional Walk (DW) have been used to detect the nodes on the ROI of the network as well as to allocate these identified holes

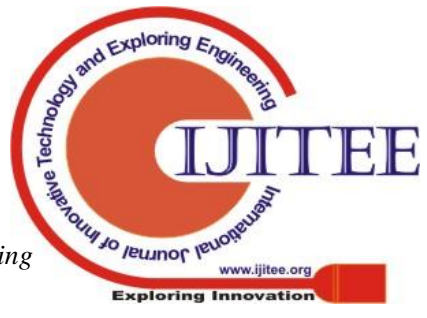


respectively. From the experiment, it has been observed that the proposed DSCS algorithm works efficiently to detect holes as well as the boundary nodes respectively. Cheng et al. (11, 2011) presented a delay aware data gathering network for WSN and helps to reduce the delay exists during the data collection process. The mainly centralized and decentralized approach has been used to design a network and concluded that these approaches help to minimize data during the data gathering process. He, Y and Q. $(12,2017)$ presented a sensor development density structure to detect and repair signal hole pair in WSN. Using this scheme the problem of coverage route as well as the hidden route can be resolved by utilizing the concept of multi-direction connected graph. Fan et al. (13, 2014) proposed an intent strategy known as 'Improved Hybrid Particle Swarm Optimization' (IHPSA) for the fixing of coverage holes. In a hybrid sensor network, the solution combines the displacement, energy consumption and energy balance of the mobile sensor nodes, effectively scheduling those nodes, repairing coverage holes by moving to the suitable location, and then increasing the event detection rate. The simulation results show that the mobile algorithm has better coverage with better event detection capability.

\section{MATERIALS AND METHODS}

The algorithm mainly goes through three different phases namely; distance calculation, node optimization and classification.

\section{A. Distance calculation}

To retrieve coverage holes, the replacement node must require determining the best distance and target position. Therefore, it becomes necessary for each sensor nodes to be determined distance so that the nodes can take a decision regarding the way where to move the node.

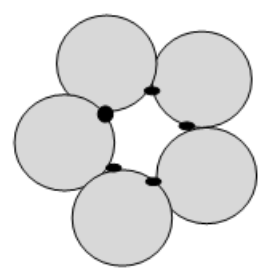

(a)

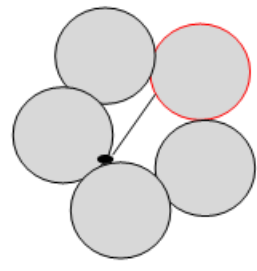

(b)
Figure 2 Intersection Point and the moving distance

These distance calculations will be used to select the most qualified replacement node. Particularly, each node calculates coordinates of the pair of intersections with each neighboring node. Using this formula, the points that are nearer to the coverage hole are considered whereas the remaining points are discarded [14].

The intersection of hole pair determined at the ROI of the network is shown in figure 2 . The distance can be calculated by using the formula written below:

$$
d=\sqrt{\left(x_{i}^{2}-x_{j}^{2}\right)}+\left(y_{i}^{2}-y_{j}^{2}\right)-r \ldots \ldots(i)
$$

In equation (i), the moving distance of sensor points is determined by distance $\mathrm{d}$, it is basically the distance between the ROI of the existing node and the point of intersection of the distant node as shown in figure 2(b) The distance of these points can be optimized by using the $\mathrm{ABC}$ algorithm the detail description is provided in the subsequent section.

\section{B. $A B C$}

It is a swarm inspired optimization scheme used to find the best points in the network. The fitness function is decided on the basis of which, it becomes able to find the best route. In 2005, Derbis Karaboga has defined the new ABC algorithm determined by the smart behavior of bees. Artificial bee colony algorithm is as simple as PSO (Particle Swarm Optimization). In ABC algorithm, the location of food is customized by the artificial bees according to the time and the bee's goal is to find out the places of food sources with high nectar amount and at last the one with the maximum nectar [15]. The algorithm of ABC is defined below:

\section{ABC algorithm \\ Load properties of nodes \\ Evaluate the fitness function of the nodes \\ Initiate the network size \\ Fitness function \\ $\left.\begin{array}{l}A B C_{\text {fit fn }}= \\ \left\{\begin{array}{c}B_{\text {cuy }} \\ B e e_{\text {on }}\end{array} \text { if } B e e_{\text {cuy }}>B e e_{\text {on }}\right.\end{array}\right\}$}

Where $A B C_{\text {fit } f n}$ the output of fitness is function and $B e e_{c u y}$ is the total bee which is called properties of nodes and $B e e_{\text {on }}$ is the threshold value of nodes

Set iteration to 1

Define the- Employed bee

-Onlookers bee and

-Scouts bee

\section{Repetition}

\section{For each employed bee}

Construct a new solution using fitness function condition

Calculate the best fit value according to the fitness function

Calculate the probability of optimal value for the solution

\section{End}

For each onlooker bee

Select a solution depending on the fitness value

Produce a new solution

Calculate the best fit value

End

If there is a redundant solution

Then replace it with a new solution

Update the best solution and store

\section{End}

Iteration=Iteration +1

Until iteration $=$ Maximum Iteration

\section{ANN}

The optimized features of the sensor nodes are provided as an input to the input layer of the ANN structure. The ANN comprises of mainly three layers: input, output and the middle layer also known as a hidden layer. This algorithm works in the same fashion as that of the human brain. The

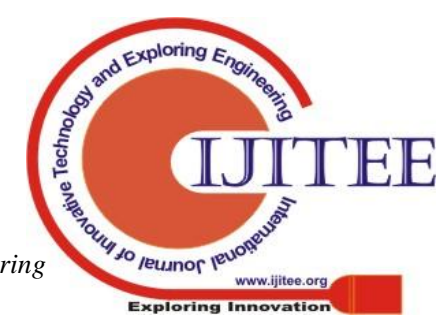


input properties such as energy consumption of node, delay, a distance of nodes are provided as an input to the input layer. In the hidden layer, the neurons are applied in such a way so that the nodes can be adjusted in such a way so that the exact solution can be determined [16]. The algorithm of ANN is defined below:

\section{ANN Algorithm:}

Input: Network properties as a Training Data (T), Target $(G)$ and Neurons (N)

Output: detect hole repairs

$$
\begin{aligned}
\text { Initialize ANN with parameters } & - \text { Epochs (E) } \\
& - \text { Neurons (N) } \\
& - \text { Performance }
\end{aligned}
$$

parameters: MSE, Gradient, Mutation \& Validation

$$
\text { - Training }
$$

Techniques: Levenberg Marquardt (Trainlm)

\section{For each set of $\mathbf{T}$}

\section{End}

$$
\text { Group }=\text { Categories of nodes }
$$

Initialized the ANN using Training data and Group

Net $=\operatorname{Newff}(T, G, N)$

Set the training parameters as per the requirements and train the WSN

Net $=$ Train (Net, Training data , Group )

Classify $=$ simulate (Net, Single node properties)

If Classify $=$ True

Genuine Node $=$ Simulated Node

Else

Affected hole repair node $=$ Simulated Node

\section{EXPERIMENTAL RESULTS}

The experiment has been performed in MATLAB simulator. The nodes range from 50 to 100 are deployed randomly in the designed network area of dimension $(1000 \times 1000)$ square meter as shown in figure 3 .

The performance of the network is measured in terms of different QoS parameters such as throughput, PDR, energy consumption, and battery usage. The throughput of the proposed network with respect to a number of iterations is shown in figure 4. The Average throughput determined for the designed network while applying $\mathrm{ABC}$ with $\mathrm{ANN}$ approach or after the detection of coverage hole is about $90.7 \%$. From the figure 5 it is clear that as the number of iterations increases throughput decreases.

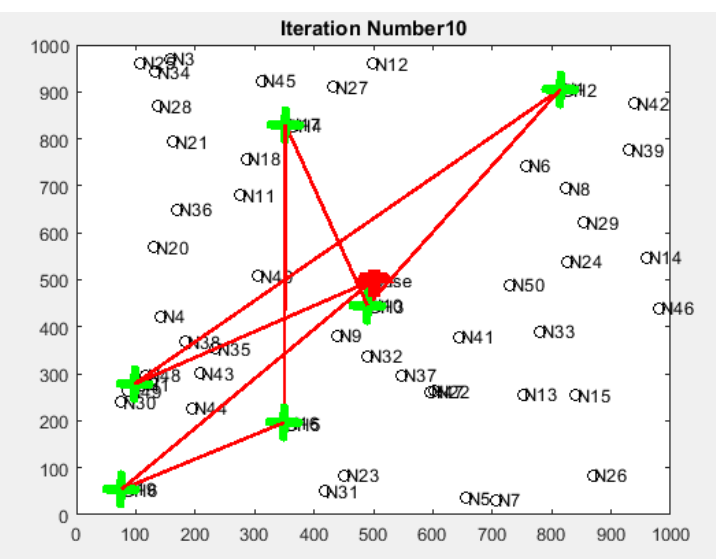

Figure 350 nodes are deployed to monitor 1000 square meter network area.

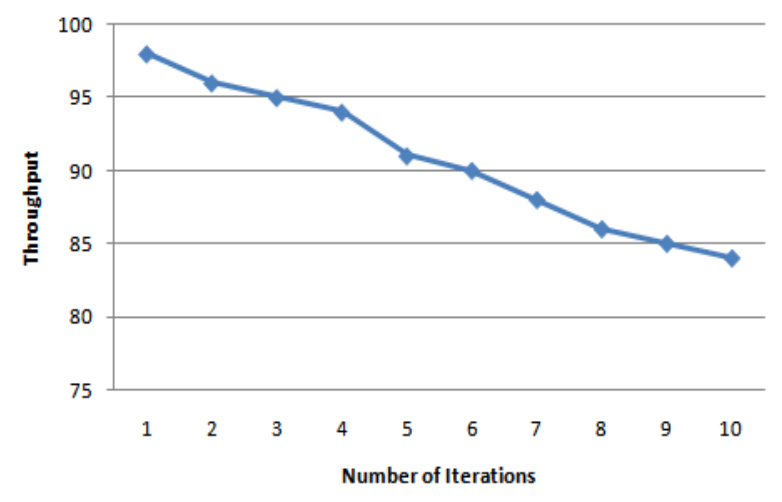

Figure4 Throughput

Packet delivery ratio represents the rate of transmitting a number data packet to the receiver with respect to the total number of data transmitted from the source node. In this research after the detection of coverage hole, the PDR of about 0.525 has been achieved.

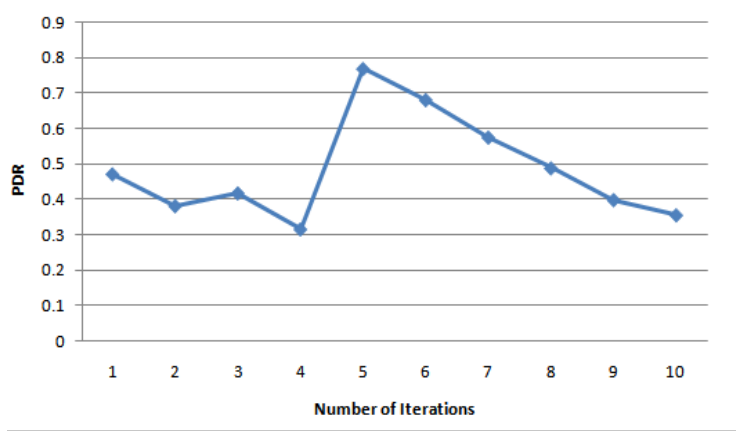

Figure5 Packet Delivery Ratio

The main focus of this research is to minimize energy consumption. Therefore, using ABC with ANN approach the energy consumed by the nodes while detecting and preventing the network from the coverage hole repair is shown in figure 6. 


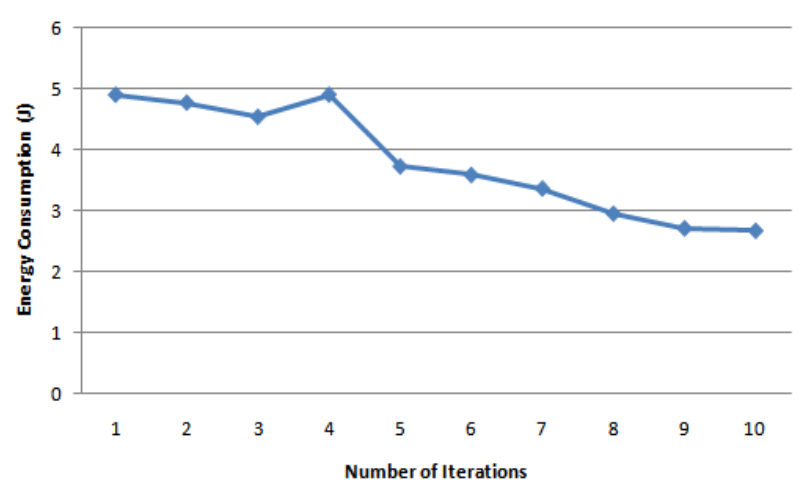

Figure6 Energy consumption (j)

The average energy consumed by the nodes of about $3.806 \mathrm{~J}$ has been obtained. To show the efficiency and the enhanced of our work, a comparison between proposed and existing work is provided as shown in figure 7 .

\section{Comparison of Energy Consumption}

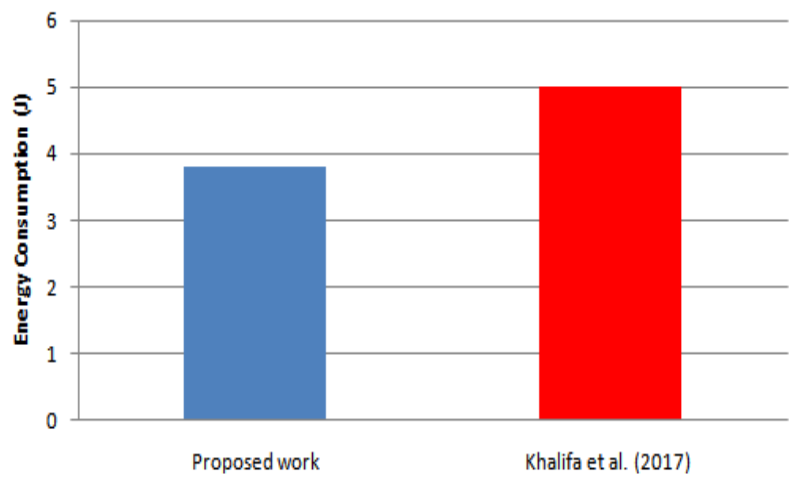

Figure 7 Comparison of energy consumption with existing work.

\section{CONCLUSION}

Depending upon the minimization concept of the coverage overlap area the network area can be maximized. Therefore by proper utilizing the concept of swarm intelligence inspired $\mathrm{ABC}$ algorithm along with machine learning technique (ANN), the connectivity among the nodes retains. The coverage hole repair algorithm is designed to maintain network coverage and connectivity through the limited mobility of nodes. The shortest distance identified by using the distance formula has been optimized using an appropriate fitness function of the $\mathrm{ABC}$ algorithm. The detection of holes has been performed using the ANN technique. Using this process, the energy up to $23.88 \%$ has been saved compared to the existing approach.

\section{REFERENCES}

1. Zhu, C., Zheng, C., Shu, L., \& Han, G. (2012). A survey on coverage and connectivity issues in wireless sensor networks. Journal of Network and Computer Applications, 35(2), 619-632.

2. Ahmed, N., Kanhere, S. S., \& Jha, S. (2005). The holes problem in wireless sensor networks: a survey. ACM SIGMOBILE Mobile Computing and Communications Review, 9(2), 4-18.

3. Tian, Y., Chang, X., Ou, Y., \& Jiang, Y. (2019, April). Coverage Hole Detection Algorithm Based on Cooperative Probability Coverage in Wireless Sensor Networks. In 2018 5th IEEE International Conference on Cloud Computing and Intelligence Systems (CCIS) (pp. 835-840). IEEE.
4. Htun, A. M., Maw, M. S., \& Sasase, I. (2014, September). Reduced complexity on mobile sensor deployment and coverage hole healing by using adaptive threshold distance in hybrid wireless sensor networks. In 2014 IEEE 25th Annual International Symposium on Personal, Indoor, and Mobile Radio Communication (PIMRC) (pp. 1547-1552). IEEE.

5. Tamboli, N., \& Younis, M. (2010). Coverage-aware connectivity restoration in mobile sensor networks. Journal of network and computer applications, 33(4), 363-374.

6. Wang, J., Ju, C., Gao, Y., Sangaiah, A. K., \& Kim, G. J. (2018). A PSO based energy efficient coverage control algorithm for wireless sensor networks. Comput. Mater. Contin, 56, 433-446.

7. Yi, L., Deng, X., Zou, Z., Ding, D., \& Yang, L. T. (2018). Confident information coverage hole detection in sensor networks for uranium tailing monitoring. Journal of Parallel and Distributed Computing, 118, 57-66.

8. Amgoth, T., \& Jana, P. K. (2017). Coverage hole detection and restoration algorithm for wireless sensor networks. Peer-to-Peer Networking and Applications, 10(1), 66-78.

9. Sahoo, P. K., \& Liao, W. C. (2015). HORA: A distributed coverage hole repair algorithm for wireless sensor networks. IEEE Transactions on Mobile Computing, 14(7), 1397-1410.

10.Zhao, L. H., Liu, W., Lei, H., Zhang, R., \& Tan, Q. (2016). Detecting boundary nodes and coverage holes in wireless sensor networks. Mobile Information Systems, 2016.

11.Cheng, C. T., Chi, K. T., \& Lau, F. C. (2011). A delay-aware data collection network structure for wireless sensor networks. IEEE sensors journal, 11(3), 699-710

12.He, Y. Q. (2017). Signal hole repair strategy based on sensor deployment density for mobile crowd network. EURASIP Journal on Embedded Systems, 2017(1), 16.

13.Fan, X., Zhang, Z., Lin, X., \& Wang, H. (2014, June). Coverage hole elimination based on sensor intelligent redeployment in WSN. In The 4th Annual IEEE International Conference on Cyber Technology in Automation, Control and Intelligent (pp. 336-339). IEEE.

14.Deng, L., Ma, X., Gu, J., \& Li, Y. (2018). DETECTION AND REPAIR OF COVERAGE HOLES IN MOBILE SENSOR NETWORKS USING SUB-VORONOI CELLS. International Journal of Robotics and Automation, 33(6).

15. Mann, P. S., \& Singh, S. (2019). Improved artificial bee colony metaheuristic for energy-efficient clustering in wireless sensor networks. Artificial Intelligence Review, 51(3), 329-354.

16. Yuan, Q., Wei, Y., Meng, X., Shen, H., \& Zhang, L. (2018). A multiscale and multidepth convolutional neural network for remote sensing imagery pan-sharpening. IEEE Journal of Selected Topics in Applied Earth Observations and Remote Sensing, 11(3), 978-989.

17.Khalifa, B., Al Aghbari, Z., Khedr, A. M., \& Abawajy, J. H. (2017) Coverage hole repair in WSNs using cascaded neighbor intervention. IEEE Sensors Journal, 17(21), 7209-7216.

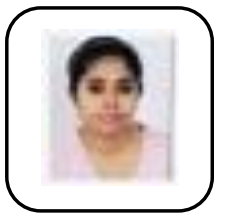

\section{AUTHORS PROFILE}

Mainka Nafri, received her Bachelor's degree in Electronics and communication Engineering (ECE) from Chandigarh University, Mohali, India in 2017. She is pursuing her Master's in Engineering in ECE from Chandigarh University, Mohali. Her research interests include Wireless Sensor Networks.

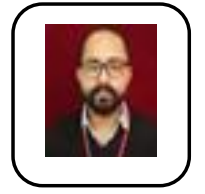

Khushal Thakur is serving as an Assistant Professor at Chandigarh University. His areas of interest are mmWaves, Massive MIMO \& Sensor Networks. He has more than four patents to his name \& has published a book on mmWaves.

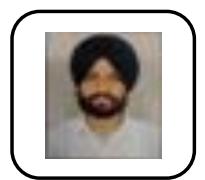

Kiran Jot Singh is a $\mathrm{PhD}$ Research Scholar in Chandigarh Univesity, Mohali, India. He has keen interest in embedded systems and robotics. He has filed 8 patents in Indian patent office.

Published By: 